\title{
Ranking Games that have Competitiveness-based Strategies*
}

\author{
Leslie Ann Goldberg ${ }^{1}$, Paul W. Goldberg ${ }^{1}$, Piotr Krysta $^{1}$, Carmine Ventre ${ }^{2}$ \\ 1 Dept. of Computer Science \\ University of Liverpool \\ Ashton Street, Liverpool L69 3BX, U.K. \\ 2 School of Computing \\ Tesside University \\ Borough Road, Middlesbrough, TS1 3BA, U. K.
}

\begin{abstract}
An extensive literature in economics and social science addresses contests, in which players compete to outperform each other on some measurable criterion, often referred to as a player's score, or output. Players incur costs that are an increasing function of score, but receive prizes for obtaining higher score than their competitors. In this paper we study finite games that are discretized contests, and the problems of computing exact and approximate Nash equilibria. Our motivation is the worst-case hardness of Nash equilibrium computation, and the resulting interest in important classes of games that admit polynomial-time algorithms. For games that have a tie-breaking rule for players' scores, we present a polynomial-time algorithm for computing an exact equilibrium in the 2-player case, and for multiple players, a characterization of Nash equilibria that shows an interesting parallel between these games and unrestricted 2-player games in normal form. When ties are allowed, via a reduction from these games to a subclass of anonymous games, we give approximation schemes for two special cases: constant-sized set of strategies, and constant number of players.
\end{abstract}

\section{Introduction}

We consider a class of finite games, and the problem of computing their exact and approximate Nash equilibria. In these games, each pure strategy of a player corresponds to a score, i.e., a level of attainment (on some measurable criterion). Each score also has an associate cost of effort (which we assume here is player-specific), where a higher scores requires higher effort. If all players select strategies, then their payoffs are obtained as follows. The players are ranked according to the scores 1 they selected, and prizes are awarded to players according to their position in the ranking. The overall payoff to a player is the value of the prize he wins, minus the cost of effort for the strategy he chose. Consequently the players face a trade-off between the cost of a strategy, and its effectiveness at winning prizes. We call these games competitiveness-based ranking games.

By way of illustration, consider a set of athletes who are training for a race. Each athlete spends time and effort in training, and has an increasing function that maps this upfront cost to

*This work was supported by EPSRC grant EP/G069239/1 "Efficient Decentralised Approaches in Algorithmic Game Theory." A preliminary version of this paper appeared as 19.

${ }^{1}$ We are following Siegel 33 in using "score" to refer to this quantity; it is also called "output" in labor-market contests ([26] and subsequent papers), or the "bid" [27]. 
performance (speed in the race). His total utility is the value of the prize that he wins, minus the cost of making this initial effort. Note that the prize is awarded based on his speed relative to other competitors, with no consideration given to his speed taken in isolation.

\subsection{Motivation}

For unrestricted normal-form games, the PPAD-completeness results of [13, 9] suggest that a Nash equilibrium is hard to compute in the worst case. Faced with a worst-case hardness result, there are two general routes to computationally positive results. We may move to the problem of computing a weaker solution concept, such as approximate Nash equilibrium (defined in detail in Section 2.2) where for some $\epsilon>0$ a player's incentive to change strategy is at most $\epsilon$. For this direction, progress has been rather limited 2 The main open problem of this line research is to determine the existence of a polynomial-time approximation scheme (PTAS); it is known from 9] that a fully polynomial-time approximation scheme (FPTAS) is as hard as computing an equilibrium exactly. The alternative route to positive results is to note that the PPAD-hardness of computing a Nash equilibrium relies on a class of highly-artificial games, suggesting that we instead focus on special cases that represent important and well-motivated games. When we find that (exact or approximate) Nash equilibria can indeed be efficiently computed for such a class of games, this overcomes the complexity-theoretic objection to using Nash equilibrium as a solution concept. Various classes of potential games, games on congestion networks, and games on graphs (representing social networks, or networks with local interactivity) do indeed have efficiently computable Nash equilibria, see [31] for an overview. The contests for prizes that we study here have a rich economics literature, but we believe that this is the first paper to analyze the problem of computing their Nash equilibria. While we find that certain algorithms for anonymous and polymatrix games are applicable to special cases, we also exhibit novel polynomial-time algorithms and approximation schemes for games in this class.

The kind of games that we study here are often constructed by a competition organizer (for example, sporting contests, or reward structures in organizations). Hence there is an associated mechanism design problem of structuring the contest in such a way as to elicit competitive behavior from the participants. Efficient computation of Nash equilibria for these games should help with the problem of determining, for example, a good allocation of prizes in such a contest. We noted that the strategies available to players have associated levels of effort and score, so in a given Nash equilibrium it is straightforward to compute, for example, the total effort or score of the players. In this way we have a well-defined measure of goodness of a Nash equilibrium.

Notice that for the players, it is socially optimal to use strategies having minimal effort, since all prizes must be allocated, irrespective of what the players have achieved (it is relative, not absolute, achievement that gets rewarded). However, in a Nash equilibrium, players will typically be more than minimally competitive. Competitiveness amongst the players results in a positive externality - in the context of spectator sports, the spectators prefer to see a well-run race, or in the context of research contests [8] (such as the DARPA Grand Challenge), competitiveness leads to research progress. We continue by reviewing the background literature in more detail.

\footnotetext{
${ }^{2}$ For polynomial-time algorithms the lowest $\epsilon$ that has been achieved for bimatrix games is just over $\frac{1}{3}$ [35], and for well-supported approximate equilibria (for which there is a further constraint that no positive probability may be allocated to any pure strategy that is worse than the best response by more than $\epsilon$ ) it is just below $\frac{2}{3}$ [18. For normal-form games with more than 2 players, known results are even weaker [5, 21. These $\epsilon$-values assume all payoffs lie in the range $[0,1]$, so that achieving $\epsilon=1$ is trivial, while $\epsilon=0$ corresponds to an exact equilibrium.
} 


\subsection{Related work}

As we noted, there is an extensive literature on contests where players exert effort with the aim of outperforming their rivals. The new aspect of this work is our focus on the design of polynomialtime algorithms for computing outcomes (equilibria) of these games. Most previous work considers continuous games, where players have a continuum of actions to choose from, with real-valued functions from effort to score. Here we study a discretized version so that we have finite games. These finite games can still serve as approximations to the continuous ones, and the resulting discretized functions benefit from a natural representation (as a list of pairs of values associating effort cost with score, for each player) so that we have a clear notion of "input size" of a problem instance, as needed in the context of polynomial-time algorithms.

The most closely-related class of finite games in the literature, appears to be the ranking games of Brandt et al. 3]. In such a game, the outcome of any pure-strategy profile is a rank-ordering of the players, and a player's utility is a decreasing function of his position in the rank-ordering. However, the games studied in [3] allow an arbitrary dependence of rankings on the pure-strategy profiles that may cause them. There is no requirement that certain pure strategies are more likely than others to raise a player's ranking. This leads to computational hardness results, notably that unrestricted ranking games are hard, even for just 3 players [3].

Anonymous games represent a class of finite games that relate to the discretized contests considered here. Anonymous games are games where a player's payoff depends on his own action and on the distribution of actions taken by the other players, but not on the identities of the players who chose each action. Anonymous games admit polynomial-time approximation schemes (PTAS's) [12, 14] but may be PPAD-complete to solve exactly. The algorithms for anonymous games can be applied to an interesting subclass of the discretized contests that we study here. In particular they apply to a special case in which all players have the same (finite) set of score levels available to them, with prizes being shared in the event of ties (which is a standard assumption in much of the literature).

We next mention some of the more classical literature on continuous contests. An influential line of work is the literature on rent-seeking problems, initiated by Tullock [36]. These are problems in which players compete to receive favorable treatment from a regulator. Another large body of literature initiated by Lazear and Rosen [26] has focused on contests in labor markets. Lazear and Rosen study the merits of rank-based prizes (as an alternative to paying a piece rate) as a means of incentivizing effort by workers in organizations. These models incorporate a random noise process that affects the selection of the winner. In a Tullock contest, the probability of winning is the amount of effort exerted by a player, divided by the total effort. Besides being a model of artificial competition, games of this kind are a model for competition for status within society [23].

Closer to the setting of this paper, are contests where the outcome is a deterministic function of effort spent by the players. Siegel [33] studies properties of the Nash equilibria of (continuous) contests, in a setting where all prizes have the same value, so that a contestant either wins or loses. He analyses expected payoffs to players, and also participation (which refers to the decision by a player to make more than a minimal expected effort). An important special case is first-price all-pay auctions, where an item (the prize) is sold to the bidder who makes the highest offer, but all bidders must pay, even if they lose. In an all-pay auction, there is a sense in which (cost of) effort is the same thing as score. Where players have different valuations for the prize, Baye et al. 1] show that this can be interpreted as score being some linear function of effort, with scaling factor proportional to the valuation. For the case of two players, Hillman and Riley [22] study the cumulative probability distributions of effort choices in the unique Nash equilibrium of the game; the uniqueness of the equilibrium is proved in [1]. The latter paper also extends the analysis to the 
case of first-price all-pay auctions with many players. A survey of results and models considered in contests (without noise) is contained in [25]. In general, our games can be viewed as a discretized version of contests without noise and could be used as an approximation to these games, provided the discretization is fine enough.

As we noted, this model has a corresponding mechanism design problem of allocating values to a set of prizes in a contest (so as to maximize total effort or score by the players). A well-known paper of Moldovanu and Sela [27] analyses this question in a setting where the players' functions (from effort to score) differ from each other in being linearly scaled by each player's ability. They obtain results for allocating value to prizes, given prior distributions over the players' abilities. ([27] also begins with an informative and readable discussion and motivation for the study of contests.) Moldovanu et al. 29] study how to elicit maximal effort via the selection of rank-based status classes, where a player gains utility from having others assigned to lower classes, and disutility from others in higher classes. (In [29] each player has a privately-known ability, relating score to cost of effort, which has been generated by a probability distribution. The solution concept is a shared function mapping ability to effort.) Their model relates to our result for linear-prize games (Section 3.3), in which the value of taking $k$-th place in the ranking is linear (decreasing) as a function of $k$; that essentially corresponds to $d$ status classes each of size $1, d$ denoting the number of players. Note that the setting we study here is different, in that players have arbitrary discretized functions from effort to score, and the functions are all commonly known. An alternative approach to maximizing effort [28] considers how to divide the competitors into sub-contests whose winners then compete in a final round.

Szymanski 34] considers the application of the theory of contests to the design of sporting competitions. The allocation of prizes in dynamic sport contests in which players determine their efforts at different stages of the game (e.g., at the beginning of each half in a soccer game) is considered in [7]. There, the focus is on comparing rank-based versus score-based prizes when spectators care about contestants' efforts or about the "suspense" of the game (see also [27, 7]). Cohen et al. [11] study a version of the contest design problem where the prize fund may be chosen by the designer, who wants to maximize the effort elicited, with prizes representing the price paid for effort. A related line of research [20, 6] has addressed from a game-theoretic perspective, the impact of the point scoring system on offensive versus defensive play in the UK Premier League; our concern here is slightly different, being focused on highly competitive versus weakly competitive play.

\subsection{Our Contribution}

Some of our algorithms apply specifically to an interesting special case of games without ties where players cannot share a prize as a result of obtaining the same score 3 For games without ties, we give an efficient algorithm for the 2-player case, and for the multi-player case we show how to compute the probabilities in a Nash equilibrium if the supports of the players' distributions are known (Theorem 2). (This shows an interesting parallel with general 2-player bimatrix games, where a Nash equilibrium having known support can be efficiently computed.) When ties are possible - in the literature, the standard assumption is that prizes are shared - it is convenient to reduce these games to equivalent score-symmetric games (Definition 2) in which all players have the same available set of score values, but with player-dependent costs. The reduction incurs an increase in the number of strategies that is proportional to the number of players. For these games,

\footnotetext{
${ }^{3}$ As an example of this consider the competition amongst universities for places in a ranking, or league table. In such a ranking, it is necessary to list the names of institutions in a strict order; ties have to be broken somehow, and this is often done alphabetically by name of institution.
} 


\begin{tabular}{|c|c|c|c|c|}
\cline { 2 - 5 } \multicolumn{1}{c|}{} & \# players & \# prizes & \# actions & Result \\
\hline \multirow{2}{*}{$\begin{array}{c}\text { Score-symmetric } \\
\text { games }\end{array}$} & any & any & $\mathrm{O}(1)$ & PTAS (Thm. 6) \\
\cline { 2 - 5 } & $\mathrm{O}(1)$ & $\mathrm{O}(1)$ & any & FPTAS (Thm. [7) \\
\cline { 2 - 5 } & any & any & 2 & Exact Pure (Thm. [5) \\
\hline Games without ties & 2 & 2 & any & Exact (Thm. [3) \\
\hline Linear-prize games & any & \# players & any & Exact (Thm. [4) \\
\hline
\end{tabular}

Table 1: Our algorithmic contributions.

a special case of interest arises when we assume a constant limit on the number of these strategies, and the results of [12, 14] can be used to provide a PTAS; here we give a simpler PTAS for such games. Table 1 gives our algorithmic results for both classes of games, together with linear-prize games (Section 3.3), a class of ranking games in which the prize for taking position $k$ in the ranking is a linear function of $k$.

\section{Model, notation and some illustrative examples}

We work in a classical game-theoretic setting of a finite number of players, each with a finite number of actions, and we consider the problem of computing Nash equilibria, and approximate Nash equilibria, for these games.

In Section 2.1 we specify in detail the class of games that we study, and introduce some notation and terminology. Section 2.2 gives the background definitions of Nash and approximate Nash equilibrium. Section 2.3 shows some examples to illustrate various technical issues.

\subsection{Definition and Notation}

A prize refers to the reward that a player gains from obtaining a specified position in the ranking, and this relates directly to the standard usage of "first prize", "second prize" etc in competitions. We say that an action is "stronger" or "more competitive" than another one, if its score is higher. Any pair of actions are comparable in this sense, whether or not they belong to the same player.

We next formally define the class of games we introduce and study in this paper. Throughout, we let $d$ denote the number of players in a game, and $n$ the number of strategies available to each player.

Definition 1 In a competitiveness-based ranking game, the $j$-th pure strategy of player $i$, denoted $a_{j}^{i}$, has associated a cost $c_{j}^{i}$ and a score $s_{j}^{i}$. We assume they are indexed in increasing order of competitiveness so that, for all $i, j$, we have $c_{j}^{i}<c_{j+1}^{i}$ and $s_{j}^{i}<s_{j+1}^{i}$. Any pure-strategy profile results in a ranking of the players according to the scores. A player whose position in the ranking is $k$ gets awarded the $k$-th prize, having value $u_{k}$. Prizes are non-increasing with respect to ranks: $u_{k} \geq u_{k+1}$, for $1 \leq k<d$, with the assumption that $u_{1}>u_{d}$ 近 In the event of a tie (where two or more players obtain the same score and are ranked equal) the prizes that would result from tiebreaking are shared. The total payoff to a player will be the value of the prize he is awarded, minus the cost of the action selected by that player.

\footnotetext{
${ }^{4}$ This assumption simply rules out an uninteresting case in which competition is not adequately incentivised. Indeed, when $u_{1}=u_{d}$, the profile in which all players play their least competitive action is a dominant strategy equilibrium.
} 
For the purpose of designing algorithms that search for a Nash equilibrium, we can assume without loss of generality that we have strict monotonicity of effort costs and scores, in the definition above. If two different actions (i) have the same cost and different scores then the stronger dominates the weaker and (ii) have the same score and different costs then the cheaper will dominate the more expensive.

Next we define an interesting subclass of the games we consider.

Definition 2 A score-symmetric game is a competitiveness-based ranking game in which all players have the same set of pure strategies (which we denote $a_{1}, \ldots, a_{n}$ ) having the same scores (which we denote $\left.s_{1}, \ldots, s_{n}\right)$. Costs remain player-specific, and $c_{j}^{i}$ denotes the cost to player $i$ of pure strategy $a_{j}$.

\subsection{Exact and approximate Nash equilibria}

Here we give the definitions of Nash equilibrium and approximate Nash equilibrium, also some further notation we use throughout. Let $S_{i}$ be the set of player $i$ 's pure strategies; $S_{i}=\left\{a_{j}^{i}\right\}_{j}$. Let $S=S_{1} \times \ldots \times S_{d}$ be the set of pure-strategy profiles, where recall $d$ denotes the number of players. It is convenient to define $S_{-i}=S_{1} \times \ldots \times S_{i-1} \times S_{i+1} \times \ldots \times S_{d}$ as the set of pure-strategy profiles of all players but $i$.

A mixed strategy for player $i$ is a distribution on $S_{i}$, that is, real numbers $x_{j}^{i} \geq 0$ for each strategy $a_{j}^{i} \in S_{i}$ such that $\sum_{j \in S_{i}} x_{j}^{i}=1$. A set of $d$ mixed strategies (one for each player) is a mixed strategy profile. By $u_{s}^{i}$ we denote the utility to player $i$ in strategy profile $s$. A mixed strategy profile $\left\{x_{j}^{i}\right\}_{j \in S_{i}}, i=1, \ldots, d$, is called a (mixed) Nash equilibrium if, for each $i, \sum_{s \in S} u_{s}^{i} x_{s}$ is maximized over all mixed strategies of $i$-where for a strategy profile $s=\left(s_{1}, \ldots, s_{d}\right) \in S$, we denote by $x_{s}$ the product $x_{s_{1}}^{1} \cdot x_{s_{2}}^{2} \cdots x_{s_{d}}^{d}$. (The notation $x_{s}$ naturally extends to strategy profiles $s \in S_{-i}$.) That is, a Nash equilibrium is a set of mixed strategies from which no player has a incentive to unilaterally deviate. It is well-known (see, e.g., [32]) that the following is an equivalent condition for a set of mixed strategies to be a Nash equilibrium:

$$
\sum_{s \in S_{-i}} u_{j s}^{i} x_{s}>\sum_{s \in S_{-i}} u_{j^{\prime} s}^{i} x_{s} \Longrightarrow x_{j^{\prime}}^{i}=0
$$

The summation $\sum_{s \in S_{-i}} u_{j s}^{i} x_{s}$ in the above equation is the expected utility of player $i$ if $i$ plays pure strategy $j$ and the other players use the mixed strategies $\left\{x_{k}^{i^{\prime}}\right\}_{k \in S_{i^{\prime}}} i^{\prime} \neq i$. Nash's theorem [30] asserts that every game has a Nash equilibrium.

We say that a set of mixed strategies $x$ is an $\epsilon$-approximately well supported Nash equilibrium, or $\epsilon$-Nash equilibrium for short, if, for each $i$, the following holds:

$$
\sum_{s \in S_{-i}} u_{j s}^{i} x_{s}>\sum_{s \in S_{-i}} u_{j^{\prime} s}^{i} x_{s}+\epsilon \Longrightarrow x_{j^{\prime}}^{i}=0 .
$$

Condition (2) relaxes that in (11) by allowing a strategy to have positive probability in the presence of another strategy whose expected payoff is better by at most $\epsilon$.

\subsection{Some examples}

We consider some examples that should be helpful in understanding the model and issues arising. Example 1 shows that the games we consider do not always have pure Nash equilibria. 
Example 1 Consider two players; for $i=1,2$ player $i$ has two actions $a_{1}^{i}$ and $a_{2}^{i}$. Suppose the row player (player 1) is stronger than the column player in the sense that the column player only wins by playing $a_{2}^{2}$ while the row player plays $a_{1}^{1}$ - this can be achieved by setting $s_{1}^{2}=2, s_{1}^{1}=3$, $s_{2}^{2}=4, s_{2}^{1}=5$. Suppose the costs are $c_{1}^{i}=0, c_{2}^{i}=\frac{1}{2}$ for both players $i=1,2$, and we have a single prize worth 1 , i.e., $u_{1}=1$ and $u_{2}=0$. We have payoff matrix:

\begin{tabular}{c|cc} 
& $a_{1}^{2}$ & $a_{2}^{2}$ \\
\hline$a_{1}^{1}$ & $(1,0)$ & $\left(0, \frac{1}{2}\right)$ \\
$a_{2}^{1}$ & $\left(\frac{1}{2}, 0\right)$ & $\left(\frac{1}{2},-\frac{1}{2}\right)$
\end{tabular}

It is easily checked that this game has no pure Nash equilibrium and that the unique equilibrium is the one in which both players mix uniformly.

Example 2] is an anonymous game with binary actions (studied in [2], although [2] studies a continuum of players). The example shows that in this kind of game, there may be multiple equilibria, and the number of equilibria may be exponential in the number of players.

Example 2 Consider a symmetric game with an even number $d \geq 4$ of players; a single prize worth 1 unit; each player $i$ has two actions $a_{1}$ and $a_{2}$ with costs $c_{1}=0$ and $c_{2}=c$ (we do not have a superscript to identify a player, since the games are symmetric). The prize will be shared between players who use $a_{2}$, or all players if they all use $a_{1}$.

Notice first that for $c \in\left(0, \frac{1}{d}\right]$, there is a pure equilibrium in which all players play $a_{2}$. For $c \in\left(\frac{1}{d}, 1\right)$ there is, by symmetry, a fully-mixed Nash equilibrium where all players play $a_{2}$ with the same probability. This can be seen by the following argument. Suppose each player, other than the first, plays $a_{2}$ with probability $p$. The first player has an incentive to play $a_{2}$ which is decreasing in $p$. In particular, for $p=1$ player 1 has no incentive to play $a_{2}$, while for $p=0$ player 1 has an incentive to play $a_{2}$. Then, by continuity, there exists a value of $p$, say $p^{*}$, for which player 1 is indifferent between $a_{1}$ and $a_{2}$. In a profile in which all players play $a_{2}$ with probability $p^{*}$ all players are indifferent by symmetry.

Now put $c=\frac{2}{d}-\epsilon$, where $\epsilon<\frac{2}{d^{2}+2 d}$. We claim that there are also pure Nash equilibria where any subset of size $\frac{d}{2}$ play pure $a_{2}$ and the others play pure $a_{1}$. A player playing $a_{2}$ obtains utility $-c+\frac{2}{d}>0$; no incentive to switch to $a_{1}$. A player playing $a_{1}$ obtains utility 0 , and by switching to $a_{2}$ would obtain utility $-c+1 /\left(\frac{d}{2}+1\right)=\epsilon-\frac{2}{d}+\frac{2}{d+2}<0$. Indeed there are also many mixed equilibria where a subset of the players play pure $a_{1}$ and the other players all use the same probabilities.

Observe that there are no Nash equilibria where players may mix with different probabilities two such players would both be indifferent between $a_{1}$ and $a_{2}$, but their expected payoffs from playing $a_{2}$ would have to differ.

The following example shows that there is no bound on the price of anarchy and on the price of stability in these games.

Example 3 Consider a symmetric game with 2 players; a single prize worth 1 unit; each player $i$ has two actions $a_{1}$ and $a_{2}$ with scores $s_{1}<s_{2}$ and costs $c_{1}=0$ and $c_{2}=1 / 2-\epsilon$, for some small $\epsilon>0$. The payoff for playing $\left(a_{2}, a_{2}\right)$ is $\epsilon$ for both players, and it is higher than the payoffs obtained by deviating from the strategy $a_{2}$ : player 1 has a payoff of 0 for strategy profile $\left(a_{1}, a_{2}\right)$ and so does player 2 for strategy profile $\left(a_{2}, a_{1}\right)$. Thus, strategy profile $\left(a_{2}, a_{2}\right)$ is a pure Nash equilibrium and its social welfare is $2 \epsilon$. Now, notice that $a_{2}$ is actually a strictly dominant strategy for both players thus implying that no other action profile is a Nash equilibrium. The action profile that maximizes the social welfare is $\left(a_{1}, a_{1}\right)$, and its social welfare is 1 . Thus, the price of anarchy in this game 
is $1 /(2 \epsilon)$. Since this Nash equilibrium is unique, $1 /(2 \epsilon)$ is also the price of stability of this game. Because $\epsilon$ can be chosen arbitrarily small, both price of anarchy and price of stability are unbounded. Note that this game is essentially the Prisoner's Dilemma in which $a_{1}$ is the collaborating strategy and $a_{2}$ is the defecting one.

\section{Algorithms and proofs}

We start by noting some preprocessing steps that establish some useful assumptions that we can make without loss of generality. We continue in Section 3.2 by considering separately the special case where players cannot tie for a position in the ranking; this case would arise in competitions that have a tie-breaking rule, or where the score values $s_{j}^{i}$ are all distinct. The reason for a focus on the tie-free case is that the analysis is simpler and the Nash equilibria turn out to have a special structure. Section 3.3 applies a result of [15] for poly matrix games, to the special case where prize values decrease linearly as a function of rank position.

In Section 3.4 we study the more general case where players may tie for a position in the ranking. We show that we can focus without loss of generality on an anonymous subclass of these games. Pure Nash equilibria of these games are studied in Section 3.4.2. In Section 3.4 .3 we give a polynomial-time approximation scheme for games with a fixed number of strategies. Finally, in Section 3.4.4 we give a fully polynomial-time approximation scheme for the case of constantly-many players.

\subsection{Preprocessing}

Results about the computation of $\epsilon$-approximate equilibria require us to assume that all payoffs in a given game lie in some fixed bounded range; usually the interval $[0,1]$ is assumed. Games whose values lie outside this this range can be have their payoffs resealed into [0,1] without affected the strategic aspects of the game. With this in mind, we resale the payoffs of an arbitrary competitiveness-based ranking game as follows. We may assume that the number of prizes is equal to $d$, the number of players. This is without loss of generality - if there are more prizes than players, then only $d$ of them can be awarded (so all but the first $d$ of them can be discarded without changing the game). Also, if there are fewer prizes than players, we can just add additional prizes of value 0 . (Thus, when we say that we have a game with only $j$ prizes, what we really mean is that $u_{j+1}=\cdots=u_{d}=0$.) The first step in the preprocessing is to ensure that $u_{d}=0$. This can be done, without changing the strategic aspect of the game, by subtracting $u_{d}$ from all prizes. Next, we ensure that $u_{1}=1$. This can be done, without changing the strategic aspect of the game, by dividing all prizes, and all costs, by $u_{1}$. Next, for each player $i$, we ensure that $c_{1}^{i}=0$. This can be done, without changing the strategic aspect of the game, by subtracting $c_{1}^{i}$ from all of the costs of player $i$. Finally, we may assume that no player has an action with a cost greater than 1 , since such an action would be dominated by $a_{1}^{i}$.

Note also that the numerical values of the scores $s_{j}^{i}$ may be modified without affecting the payoffs and Nash equilibria of the game, provided only that the modification does not affect which are greater than which (in which case the ranking of the players is preserved). However, it is usually convenient to specify numerical $s_{j}^{i}$ values (rather than, more abstractly, their ordinal relationships) when describing a game.

Finally, we establish a useful fact that will be used to obtain polynomial-time algorithms that return approximate Nash equilibria. 
Observation 1 For any $\epsilon>0$ inverse of an integer, given a probability vector $\mathbf{x}=\left(x_{1}, \ldots, x_{n}\right)$, it is possible to define a probability vector $\tilde{\mathbf{x}}=\left(\tilde{x}_{1}, \ldots, \tilde{x}_{n}\right)$, called an " $\epsilon$-rounding" of $\mathbf{x}$, in which

1. each entry is equal to a non-negative integer multiple of $\epsilon$, and

2. For every $j \in\{1, \ldots, n\}$, the rounding error $\left|\sum_{k=1}^{j}\left(\tilde{x}_{k}-x_{k}\right)\right|$ is less than $\epsilon$.

Proof. We consider the values $x_{1}, \ldots, x_{n}$ in order. When we consider $x_{j}$, we round it to define $\tilde{x}_{j}$. If $x_{j}$ is an integer multiple of $\epsilon$, then $\tilde{x}_{j}=x_{j}$. Otherwise, we set the value of $\tilde{x}_{j}$ by rounding $x_{j}-$ we round up to the nearest integer multiple of $\epsilon$ if $\sum_{k<j} \tilde{x}_{k} \leq \sum_{k<j} x_{k}$ and we round down to the nearest integer multiple of $\epsilon$ otherwise. This ensures that $\left|\sum_{k=1}^{j}\left(\tilde{x}_{k}-x_{k}\right)\right|<\epsilon$.

We now show that $\tilde{\mathbf{x}}$ is a probability vector, that is, that $\sum_{k=1}^{n} \tilde{x}_{k}=1$. Now since $\mathbf{x}$ is a probability vector,

$$
\left|\left(\sum_{k=1}^{n} \tilde{x}_{k}\right)-1\right|=\left|\sum_{k=1}^{n}\left(\tilde{x}_{k}-x_{k}\right)\right|,
$$

and we already know that the latter is less than $\epsilon$. Since the $\tilde{x}_{k}$ 's are non-negative integer multiples of $\epsilon$ then $\sum_{k} \tilde{x}_{k}=a \epsilon$, for some integer $a$. The above inequality then yields $-1<a-\frac{1}{\epsilon}<1$. Since $\frac{1}{\epsilon}$ and $a$ are integer numbers, we can only satisfy the previous inequality by having $\sum_{k} \tilde{x}_{k}=1$.

\subsection{Games without ties with a single prize}

We begin by observing that (subject to the above preprocessing) we may restrict our attention to actions' costs that are strictly less than 1.

Observation 2 Assume player $i$ has an action $a_{j}^{i}$ such that $c_{j}^{i}=1$. Then $a_{j}^{i}$ is weakly dominated by $a_{1}^{i}$. Therefore, we can eliminate $a_{j}^{i}$ from the game at the price of eliminating some potential Nash equilibria.

Assuming that costs are strictly less than 1, we show that Nash equilibria of games without ties have a nice structure when there is a single prize. Siegel [33] has shown a more general version of the following, in the context of continuous games. We include a proof here, since it is simpler in the discrete case.

Theorem 1 Suppose there is a single prize of value 1 and actions' costs are less than 1 . If no two actions have the same strength (thus ties are impossible) then in any Nash equilibrium

1. There is just one player with positive expected payoff; all others have expected payoff zero.

2. The player with positive expected payoff is the one with the strongest action with a cost of less than 1.

Proof. Given the preprocessing steps noted above and the assumption that the single prize has value 1 , the costs of all actions lie in the range $[0,1)$, and each player has an action with cost 0 . Let $\mathcal{N}$ be a Nash equilibrium. For each player $i$ let $w_{i}$ be the weakest action of $i$ that lies in the support of $\mathcal{N}$; thus, $i$ has positive probability of using $w_{i}$, and all other actions that $i$ uses with positive probability are stronger than $w_{i}$.

For any action $a$, let $s(a)$ denote the score of that action. Let $p$ be the player whose weakest action in the support of $\mathcal{N}$ is stronger than all other players' weakest actions in the support of $\mathcal{N}$, thus $s\left(w_{p}\right)>s\left(w_{p^{\prime}}\right)$ for all $p^{\prime} \neq p$. 
Note that for any player $p^{\prime} \neq p$, the expected payoff to $p^{\prime}$ from using action $w_{p^{\prime}}$ is non-positive: $w_{p^{\prime}}$ cannot win since $p$ is certain to play a stronger action. But, $p^{\prime}$ gives positive probability to $w_{p^{\prime}}$, so no other action available to $p^{\prime}$ can have higher expected payoff. $p^{\prime}$ has non-positive expected payoff, and under the assumption (that we may adopt from preprocessing) that players all have a 0 -cost action, $p^{\prime}$ 's expected payoff must in fact be zero.

For the second part of the theorem, note that we have seen that all but one player must have expected payoff 0 , in Nash equilibrium $\mathcal{N}$. Let $p^{\prime \prime}$ be the player with the strongest action having a cost of less than 1 . Then $p^{\prime \prime}$ can always guarantee a positive expected payoff by using that action, so in any Nash equilibrium, must receive positive expected payoff. Hence $p^{\prime \prime}$ is the only player with non-zero expected payoff in $\mathcal{N}$ (and $p^{\prime \prime}$ is in fact the same as the above player $p$ ).

The following theorem also shows how a Nash equilibrium may be efficiently computed for tiefree games, provided that we know the support of the Nash equilibrium. This shows an interesting parallel between these games, and general 2-player normal form games, especially in conjunction with the subsequent observation that the solution is a rational number.

Theorem 2 For games with any number of players, pure-strategy costs less than 1 and a single prize where ties are impossible, a Nash equilibrium can be computed in polynomial time if we are given the support of a solution.

Proof. Given a game $\mathcal{G}$, suppose that we remove the pure strategies that are not in the support of some (unknown) Nash equilibrium. The resulting game $\mathcal{G}^{\prime}$ has a fully-mixed equilibrium $\mathcal{N}$, thus any two strategies that belong to a player have the same expected payoff in $\mathcal{N}$. Our general approach is to compute the probabilities $x_{j}^{i}$ in descending order of strength of the associated actions $a_{j}^{i}$.

Let $a_{j}^{i}$ be the strongest action in $\mathcal{G}^{\prime}$ (i.e. having the highest score). Player $i$ 's expected payoff is $1-c_{j}^{i}$ and by Theorem 1 all other players have expected payoff 0 .

Let $a_{j^{\prime}}^{i^{\prime}}$ be the second-strongest action in $\mathcal{G}^{\prime}$; we may assume $i^{\prime} \neq i$ since if $i^{\prime}=i$ then $a_{j}^{i}$ would be strictly dominated by $a_{j^{\prime}}^{i^{\prime}}$. Its expected payoff to $i^{\prime}$ is $-c_{j^{\prime}}^{i^{\prime}}+\left(1-x_{j}^{i}\right)$, which by Theorem 1 is 0 , so we have an expression for $x_{j}^{i}$. Consider the third-strongest action $a_{j^{\prime \prime}}^{i^{\prime \prime}}$, whose payoff is given by $-c_{j^{\prime \prime}}^{i^{\prime \prime}}+\left(1-x_{j}^{i}\right)\left(1-x_{j^{\prime}}^{i^{\prime}}\right)$ (assuming $i^{\prime \prime} \neq i$ ) which gives us an expression for $x_{j^{\prime}}^{i^{\prime}}$.

Generally, the $r$-th strongest action $a_{\beta}^{\alpha}$ has expected payoff $-c_{\beta}^{\alpha}+\prod_{k \neq \alpha}\left(1-\sigma_{k}\right)$ where $\sigma_{k}$ is the sum of probabilities of player $k$ 's actions that are stronger than $a_{\beta}^{\alpha}$.

The probabilities for each player's weakest actions will be obtained from the equations that ensure that for every player $i$, the values $x_{j}^{i}$ sum to 1 (are a probability distribution).

Observation 3 For games where ties are impossible, if all action costs are rational numbers smaller than 1 then the solution is also a rational number.

This is immediate from the expressions in the above proof that give the values $x_{j}^{i}$.

\subsubsection{Solving 2-player games exactly}

Ranking games (as in [3]) with actions that do not have the upfront $\operatorname{costs} c_{j}^{i}$ we consider here, are constant-sum, so they can be solved efficiently in the 2-player case. Our games are not constantsum, but we do have an alternative polynomial-time algorithm to solve them in the 2-player case.

Theorem 3 2-player ranking games that have competitiveness-based strategies and are without ties can be solved exactly in polynomial time. 
Proof. As before, assume a single prize of 1 unit and action costs in $[0,1]$, which may be assumed by the preprocessing noted earlier.

We can (in polynomial time) compute exact solutions of 2-player games of this type as follows. We start by eliminating certain dominated strategies. Specifically, suppose that for strategies $a_{j}^{i}$ and $a_{j+1}^{i}$, the set of opponent's strategies that they win against, is the same. Then $a_{j+1}^{i}$ can be eliminated. Rename the strategies of this game $\left(a_{1}, \ldots a_{n}\right)$ for the row player and $\left(a_{1}^{\prime}, \ldots a_{n^{\prime}}^{\prime}\right)$ for the column player. Assume without loss of generality that it is the row player who has the weakest strategy, thus $a_{1}^{\prime}$ wins against $a_{1}$. When $n=n^{\prime}$, the strategies, arranged in ascending order of strength are $a_{1}, a_{1}^{\prime}, a_{2}, a_{2}^{\prime}, \ldots a_{n}, a_{n}^{\prime}$. When instead $n \neq n^{\prime}$, then it must be the case that the same player has weakest and strongest strategy; the strategies, arranged in ascending order of strength, are $a_{1}, a_{1}^{\prime}, \ldots, a_{n}, a_{n}^{\prime}, a_{n+1}$ in this case.

Suppose that in some Nash equilibrium $\mathcal{N}$ the row player does not use strategy $a_{j}$ for some $j>1$ (that is, the player plays $a_{j}$ with probability 0 ). Then the column player does not use strategy $a_{j}^{\prime}$ (which is the cheapest one that wins against $a_{j}$ ) since $a_{j}^{\prime}$ would now be dominated by $a_{j-1}^{\prime}$. For a similar reason, the row player will not use $a_{j+1}$, the cheapest strategy that wins against $a_{j}^{\prime}$, so the column player will not use $a_{j+1}^{\prime}$, and so on. This shows that (in a Nash equilibrium) the strategies in either player's support must be either a prefix of the sequence of his strategies or a prefix of all his strategies but the weakest, with strategies arranged in ascending order of strength.

We can now try to solve for all such supports, since there are polynomial-many of them. Recall that a 2-player game can be solved efficiently in polynomial time if we are told the support of a solution, since it reduces to a linear program (see, for example, page 31 in [31]).

The main property used to show Theorem 3 above (i.e., if in any solution a player does not play a certain strategy $s$ then the other one does not play the strategy that "just beats" $s$ ) breaks down when ties are allowed. Simply consider two consecutive strategies of player $1, a_{i}^{1}$ and $a_{i+1}^{1}$, and of player $2, a_{j}^{2}$ and $a_{j+1}^{2}$ such that scores of $a_{i}^{1}$ and $a_{j}^{2}$ (resp. $a_{i+1}^{1}$ and $a_{j+1}^{2}$ ) are the same. In this case, we can no longer conclude that in any solution if player 1 does not play $a_{i}^{1}$ then player 2 does not play $a_{j+1}^{2}$ as such a strategy is used not just to beat $a_{i}^{1}$ but also to share with $a_{i+1}^{1}$. This shows that for games where ties may occur, we have to use different approaches to obtain polynomial-time algorithms for them.

\subsection{Exact algorithm for linear-prize ranking games}

Consider a $d$-player $n$-strategies-per-player ranking game $\mathcal{G}$ with competitiveness-based strategies without ties in which the prize for ranking $k$-th is a linear function $a-k b$, for some values $a$ and b. We call $\mathcal{G}$ a linear-prize ranking game. We claim that we can represent $\mathcal{G}$ as a poly matrix game [15. A poly matrix game can be represented as a graph: players are the verticals and a player's payoff depends on the actions of his neighbors. The edges are 2-player zero-sum games. Once all players have chosen a strategy, the payoff of each player is the sum of the payoffs of the games played with his neighbors. Dashikis and Papadimitriou [15] give an algorithm which solves polymatrix games in polynomial time.

We can express $\mathcal{G}$ as a polymatrix game as follows. We define a complete $(d+1)$-vertex graph where the additional vertex encodes an external player $N$, which we call "nature". For players $i$ and $i^{\prime}$ in $\mathcal{G}$, edge $\left(i, i^{\prime}\right)$ is an $n \times n 2$-player constant-sum game. (This is a zero-sum game, shifted by a constant - this shifting can be accommodated in polymatrix games.) The game on edge $\left(i, i^{\prime}\right)$ "punishes" the lowest-ranked player amongst $i$ and $i^{\prime}$. In particular, in the matrix of this game, the entry $\left(j, j^{\prime}\right)$ will have payoff 0 for player $i$ and $-b$ for player $i^{\prime}$ if and only if $s_{j}^{i}>s_{j^{\prime}}^{i^{\prime}}$. Edge $(i, N)$ is an $n \times 12$-player zero-sum game in which nature "gains" what player $i$ is paying in effort 
minus $a-b$. In particular, each entry $j$ will have payoff $a-b-c_{j}^{i}$ for player $i$ and payoff $c_{j}^{i}-a+b$ for nature. Note that once all players of $\mathcal{G}$ have chosen strategies, the payoff to the player who is ranked $k$-th is $a-k b$ minus his cost. He loses $b$ to each of the $k-1$ players who beats him and he gains $a-b$ and loses his cost of effort, to nature. Thus, using the algorithm in [15], we obtain the following theorem.

Theorem 4 There is a polynomial-time algorithm that computes a Nash equilibrium for linearprize ranking games.

\subsection{Games where ties are possible}

We consider a more general situation in which two or players may have pure strategies having the same score, and if they play those strategies, any prizes are shared. We begin by showing that we can study without loss of generality Nash equilibria of competitiveness-based ranking games in which scores are symmetric. That is, players have a shared set of $n$ pure strategies, $a_{1}, \ldots, a_{n}$. As above, player $i$ has player-dependent $\operatorname{costs} c_{1}^{i}<\ldots<c_{n}^{i}$, but the scores are player-independent, and we denote them $s_{1}<\ldots<s_{n}$.

\subsubsection{Reduction to score-symmetric games}

The reduction preserves Nash equilibria of the original game (and thus is a Nash homomorphism) and is presented for the case of 2-player games. The generalisation to the $d$-player case is straightforward, although the number of strategies per player would increase by a factor of $d$.

Suppose that player 1 has action $a_{j}^{1}$ and player 2 has no action with score equal to $s_{j}^{1}$. We give player 2 a weakly dominated strategy with score $s_{j}^{1}$ - if $a_{k}^{2}$ is the weakest strategy of player 2 that has higher score than $a_{j}^{1}$, give player 2 an additional strategy with $\operatorname{cost} c_{k}^{2}$ and score $s_{j}^{1}$. If player 2 does not have a stronger strategy than $a_{j}^{1}$, give player 2 an additional strategy with cost 1 and score $s_{j}^{1}$.

We can assume that each player has $n$ strategies $a_{1}, \ldots, a_{n}$ with scores $s_{1}, \ldots, s_{n}$ and costs $c_{1}^{1}, \ldots, c_{n}^{1}$ for player 1 and $c_{1}^{2}, \ldots, c_{n}^{2}$ for player 2. Suppose we solve this game, and now we have to recover a solution to the original game before the weakly dominated strategies were added. To do this, each player just has to replace their usage of any weakly dominated strategy by the corresponding weakly dominating one. Let $a_{j}$ be a weakly dominated action of player $1 ; a_{k}$ denotes the corresponding weakly dominating action, $k>j$ and then $s_{k}>s_{j}$. The probability of player 1 playing $a_{j}$ at equilibrium is then added to his probability of playing $a_{k}$. This raises the question of whether player 2 may be given an incentive to deviate as a consequence. Such an incentive can only concern actions with scores within the interval $I:=\left[s_{j}, s_{k}\right]$ (for actions with score outside $I$, player 2 has the same payoff when player 1 plays either $a_{j}$ or $a_{k}$ since in these cases the ranking of the players do not change). First, note that if in the Nash equilibrium player 2 plays actions with scores in $I$ with positive probability then player 1 plays $a_{j}$ with probability 0 . This is because player 1 strictly prefers $a_{k}$ to $a_{j}$ when player 2 plays actions with score in $I$ and is indifferent between them for the remaining actions. Therefore, we do not need to redistribute probability mass in this case. On the other hand, whenever in the Nash equilibrium the actions having score in $I$ are played by player 2 with zero probability then the change of the probability distribution of player 1 has no effect on player 2; as observed above, for each action with score outside $I$, player 2 has the same payoff when player 1 plays either $a_{j}$ or $a_{k}$ and then he has no incentive to deviate. 


\subsubsection{Score-symmetric games and pure equilibria}

Unlike games without ties, for which 2-player 2-action games might not possess pure equilibria (see Example 1), score-symmetric games in which players have only 2 strategies do have pure Nash equilibria (for any number of players and any number of prizes).

Theorem 5 2-action competitiveness-based score-symmetric ranking games do have pure Nash equilibria (any number of players; any action costs for individual players). Furthermore, a pure Nash equilibrium can be found in polynomial time.

Proof. We have 2 pure strategies $a_{1}$ and $a_{2}$, where $a_{1}$ is less competitive; thus for each player $i$ we have $c_{1}^{i} \leq c_{2}^{i}$. Recall that by preprocessing we may assume that $c_{1}^{i}=0$, so that $c_{2}^{i}$ is non-negative, for all $i$. We show how to identify a pure Nash equilibrium that consists of a (potentially empty) set of players playing $a_{2}$, all of whom have a cost for playing $a_{2}$ lower than the ones playing $a_{1}$.

We may assume that the players are indexed in non-decreasing order of their cost of playing $a_{2}$, so that for $1 \leq i<n$ we have $c_{2}^{i} \leq c_{2}^{i+1}$. Now, let $\mathcal{Z}_{i}$ denote the pure profile in which the first $i$ players play $a_{2}$ and the remaining $d-i$ players play $a_{1}$. We claim that if $i$ has an incentive to deviate from $\mathcal{Z}_{i-1}$ then no player $i^{\prime}$ has an incentive to deviate from $\mathcal{Z}_{i}$, with $i^{\prime}<i$. Indeed, if $i$ has an incentive to deviate from $\mathcal{Z}_{i-1}$, then $\frac{u_{i}+\ldots+u_{d}}{d-i+1}$, his utility in $\mathcal{Z}_{i-1}$, is strictly less than $\frac{u_{1}+\ldots+u_{i}}{i}-c_{2}^{i}$, his utility in $\mathcal{Z}_{i}$. Since, by definition, $c_{2}^{i^{\prime}} \leq c_{2}^{i}$, for $i^{\prime}<i$, the previous inequality implies that player $i^{\prime}$ is better off by sticking to $a_{2}$ in $\mathcal{Z}_{i}$. Therefore, starting from the profile $\mathcal{Z}_{0}$ in which all players play $a_{1}$, we initially check whether player 1 has an incentive to deviate. If not, the profile is a pure Nash equilibrium, otherwise we let him deviate and we have the profile $\mathcal{Z}_{1}$. We can reiterate this process for each player $i$ in this ordering until we reach the break-even point at which the share of the prize obtained from playing $a_{2}$ goes down below the cost of the next player in line. This shows the existence of the claimed pure Nash Equilibrium, and also constitutes an efficient algorithm for finding it.

A related result is known for symmetric games in which players have only 2 strategies: These games always have a pure Nash equilibrium [10. The above theorem concerns games that are anonymous but not symmetric (as costs are player-specific). However, let us notice that the arguments used in [4] to prove the result about symmetric games appear to be similar to ours. It is easy to see that these arguments fail when two players have three strategies available, as shown by the next example.

Example 4 We have 2 players and 3 actions, namely $a_{1}, a_{2}$ and $a_{3}$ ordered increasingly by score, i.e., $s_{1}<s_{2}<s_{3}$. The prizes are $u_{1}=1$ and $u_{2}=0, u_{3}=0$. Costs are $c_{1}^{i}=0$ for $i=1,2$, $c_{2}^{1}=\frac{2}{3}, c_{3}^{1}=\frac{4}{5}$ and $c_{2}^{2}=\frac{1}{3}, c_{3}^{2}=\frac{2}{3}$. Thus, we have payoff matrix

\begin{tabular}{c|ccc} 
& $a_{1}$ & $a_{2}$ & $a_{3}$ \\
\hline$a_{1}$ & $\left(\frac{1}{2}, \frac{1}{2}\right)$ & $\left(0, \frac{2}{3}\right)$ & $\left(0, \frac{1}{3}\right)$ \\
$a_{2}$ & $\left(\frac{1}{3}, 0\right)$ & $\left(-\frac{1}{6}, \frac{1}{6}\right)$ & $\left(-\frac{2}{3}, \frac{1}{3}\right)$ \\
$a_{3}$ & $\left(\frac{1}{5}, 0\right)$ & $\left(\frac{1}{5},-\frac{1}{3}\right)$ & $\left(-\frac{3}{10},-\frac{1}{6}\right)$
\end{tabular}

It is easily checked that this game has no pure Nash equilibrium. The unique Nash equilibrium of the game is $\left(\frac{2}{3}, 0, \frac{1}{3}\right)$ for player 1 and $\left(\frac{2}{5}, \frac{3}{5}, 0\right)$ for player 2 . 


\subsubsection{PTAS for many players who share a fixed set of strategies}

Consider a score-symmetric $d$-player game, each of whom have actions $a_{1}, \ldots, a_{n}$ with scores $s_{1}, \ldots, s_{n}$. In this section we view the number of actions $n$ as a constant, and we are interested in algorithms whose runtime has polynomial dependence on $d$, the number of players. A scoresymmetric game is a special case of an anonymous game, so it is possible to directly apply a result of Daskalakis and Papadimitriou [14] to show that it has a PTAS. Here, we give a conceptually simpler PTAS.

In the PTAS, we first round the cost vectors of the players. This does not introduce much error, but it ensures that there are only a constant number of different cost vectors. We refer to the cost vector of a player as its "type". Now the point is that players of the same type are equivalent in the following sense - once we know how many players of each type adopt each (mixed) strategy, we can examine the resulting strategy profile to check whether it is an $\epsilon$-Nash equilibrium. It is only important how many players of each type adopt a particular strategy - it is not important which players they are. Thus, brute-force search is quite efficient. Technically this algorithm is oblivious in the sense of Daskalakis and Papadimitriou [16], in that it constructs a polynomial-sized set of mixed-strategy profiles in such a way that at least one of them should be an approximate equilibrium, and checks each of them.

By a $k$-composition of a positive integer $N$, we mean a solution to $N_{1}+\ldots+N_{k}=N$ in which $N_{1}, \ldots, N_{k}$ are non-negative integers. There are less than $N^{k}$ such solutions.

Algorithm 1: PTAS for score-symmetric $d$-player games having a constant number, $n$, of pure strategies and a given accuracy parameter $\epsilon>0$

1 Let $\delta=\epsilon / n$ and $\ell=\lceil 1 / \epsilon\rceil$.

2 For each player, round each $\operatorname{cost} c_{j}^{i}$ down to the nearest non-negative integer multiple of $\epsilon$. Two players have the same type if they have the same set of rounded costs. Let $\mathcal{T}$ be the set of types; note that $|\mathcal{T}| \leq(\ell+1)^{n}$.

3 Let $S$ be the set of $n$-dimensional probability vectors $\left\{\left(x_{1}, \ldots, x_{n}\right)\right\}$ in which each $x_{j}$ is a non-negative integer multiple of $\delta$. (Mixed strategies for individual players will be sought from elements of $S$.) Let $s=|S|$, and note that $s \leq(n \ell+1)^{n}$.

4 Perform a brute-force search as follows. For every type $t \in \mathcal{T}$, consider every $s$-composition $d_{t}=d_{t, 1}+\cdots+d_{t, s}$ of the $d_{t}$ players of type $t$. Consider the strategy profile in which, for all $t \in \mathcal{T}$ and all $j \in\{1, \ldots, s\}, d_{t, j}$ players of type $i$ play the $j$-th strategy in $S$. Check whether this strategy profile is an $\epsilon$-Nash equilibrium.

5 Return an $\epsilon$-Nash equilibrium if one is found.

Theorem 6 For any constant $\epsilon>0$ and any constant $n$, Algorithm 1 returns a $2 \epsilon$-Nash equilibrium in time polynomial in $d$.

Proof. It is convenient to assume in the proof that $\epsilon$ (and hence $\delta$ ) is the inverse of an integer. This can be assumed by rounding $\epsilon$ down to the nearest such fraction $\epsilon^{\prime}$, obtaining an $\epsilon^{\prime}$-Nash equilibrium, which is consequently an $\epsilon$-Nash equilibrium.

Consider first the case in which $\epsilon$ is the inverse of an integer. Since costs lie in the range $[0,1]$ we can see that after Step 2 is executed there are less than $(\ell+1)^{n}$ distinct player types. We will find an $\epsilon$-Nash equilibrium of the rounded game, which is a $2 \epsilon$-Nash equilibrium of the original game since each cost is only changed by at most an additive $\epsilon$. Note that $|\mathcal{T}|=O(1)$ as a function of $d$, the number of players. Also, the number $s$ of strategies in $S$ constructed in Step 3 
is $O(1)$ as a function of $d$. Since the number of $s$-compositions of $d_{t}$ (Step 4) is $O\left(\left(d_{t}\right)^{s}\right)$, the total number of mixed-strategy profiles considered in Step 4 is at most $O\left(\prod_{t \in \mathcal{T}}\left(d_{t}\right)^{s}\right)=O\left(d^{s|\mathcal{T}|}\right)$ which is polynomial in $d$.

We now show that the algorithm always finds an $\epsilon$-Nash equilibrium in the last step. First, consider the game with rounded cost vectors, as constructed in Step 2, and let $\mathcal{N}$ be a Nash equilibrium of this game. Consider a $\delta$-rounding (as from Observation 1) of each probability vector of $\mathcal{N}$ and notice that such a probability vector is checked by the algorithm (or an equivalent one is, in which the identities of players of the same type are swapped). We next show that such a probability vector is an $\epsilon$-Nash equilibrium. A player can be playing an action with a probability that differs by at most $\delta$ from the probability he should have used for his best response. Thus for each of his actions, he can lose at most $\delta$ times the payoff he gets for that action. Since payoffs are upper-bounded by 1 , the maximum regret is at most $n \delta=\epsilon$. The proof concludes by noting that the cost rounding of Step 2 implies an extra additive error of at most $\epsilon$.

\subsubsection{FPTAS for constant number of players, many strategies}

Let $\mathcal{G}$ be a score-symmetric game with $d$ players having access to $n$ (shared) pure strategies. In this section we view the number of players $d$ as a constant, and the number of pure strategies $n$ is the parameter that governs the size of a game. Let $\{1, \ldots, d\}$ denote the players. We have $d$ prizes of values $u_{1} \geq u_{2} \geq \cdots \geq u_{d}$, where $u_{1}=1$ and $u_{d}=0$.

In this case, the expected payoff of player $i$ from playing $a_{j}$ is given by the expected prize he gets minus his cost $c_{j}^{i}$ of $a_{j}$. To define the expected prize that the player gets by playing $a_{j}$ we need some notation. Let $B=\{-1,0,1\}$ and $v=\left(v_{1}, v_{2}, \ldots, v_{d}\right) \in B^{d}$ be a vector that is defined with reference to some $a_{j}$ as follows. $v_{k}$ gives information about the pure strategy played by player $k \in\{1, \ldots, d\}$, where $v_{k}=0$ means that player $k$ plays $a_{j}, v_{k}=-1$ means that player $k$ plays one of the actions that are less competitive than $a_{j}$ (that is, one of $a_{1}, \ldots, a_{j-1}$ ), and finally, $v_{k}=1$ means that $k$ plays one of the more competitive actions than $a_{j}$ (one of $a_{j+1}, \ldots, a_{n}$ ). Given vector $v \in B^{d}$ we let $v(1)$ be the number of 1 's in $v$, i.e., $v(1)=\left|\left\{v_{i}: v_{i}=1\right\}\right|$; similarly, $v(0)(v(-1)$, respectively) denotes the number of 0's (-1's, respectively) in $v$; thus, $v(-1)+v(0)+v(1)=d$.

Now, let $x_{j}^{i_{1}}$ be the probability that player $i_{1}$ plays $a_{j}$, and let $i_{2}, \ldots, i_{d}$ be the remaining players. Observe that, if player $i_{1}$ plays purely $a_{j}$, i.e., $v_{1}=0$, then prizes $u_{1}, u_{2}, \ldots, u_{v(1)}$ will be given to $v(1)$ players playing more competitive actions than $a_{j}$, and prizes $u_{q+1}, u_{q+2}, \ldots, u_{d}$ are reserved for the $v(-1)$ players playing less competitive actions than $a_{j}$, where $q=d-v(-1)$. Thus, the total value of prizes to be shared among the $v(0)$ players playing $a_{j}$ (including player $i_{1}$ ) is $u_{v(1)+1}+u_{v(1)+2}+\cdots+u_{q}$.

We also denote by $p_{j}^{i}(-1)=\sum_{\ell=1}^{j-1} x_{\ell}^{i}$ and $p_{j}^{i}(1)=\sum_{\ell=j+1}^{n} x_{\ell}^{i}$, the probabilities of player $i$ playing actions that are less (respectively, more) competitive than $a_{j}$; furthermore, let $p_{j}^{i}(0)=x_{j}^{i}$. To cover degenerate cases we also assume that $p_{1}^{i}(-1)=0$, i.e., the probability of $i$ playing an action less competitive than $a_{1}$ (the weakest action) is zero; similarly, $p_{n}^{i}(1)=0$. Then the expected payoff of player $i_{1}$ for playing $a_{j}$, denoted as $\pi_{j}^{i_{1}}$, is given by

$$
\pi_{j}^{i_{1}}=-c_{j}^{i_{1}}+\sum_{v=\left(v_{1}=0, v_{2}, \ldots, v_{d}\right) \in\{0\} \times B^{d-1}} \frac{u_{v(1)+1}+u_{v(1)+2}+\cdots+u_{q}}{v(0)} \cdot\left(\prod_{k=2}^{d} p_{j}^{i_{k}}\left(v_{k}\right)\right) .
$$

To compute a Nash equilibrium, we need to find real values $x_{1}^{i}, \ldots, x_{n}^{i}$, for $i \in\{1,2, \ldots, d\}$, that satisfy

$$
x_{j}^{i} \geq 0 \forall i, j ; \quad \sum_{j} x_{j}^{i}=1 \quad i \in\{1,2, \ldots, d\}
$$


saying that for $i \in\{1,2, \ldots, d\}$, the values $\left\{x_{j}^{i}\right\}_{j}$ are a probability distribution; for $i \in\{1,2, \ldots, d\}$ and $j>1$ the following should also hold:

$$
\begin{aligned}
& \pi_{j}^{i}>\max _{k=1, \ldots, j-1}\left\{\pi_{k}^{i}\right\} \quad \Longrightarrow x_{1}^{i}=\ldots=x_{j-1}^{i}=0 \\
& \pi_{j}^{i}<\max _{k=1, \ldots, j-1}\left\{\pi_{k}^{i}\right\} \quad \Longrightarrow x_{j}^{i}=0 .
\end{aligned}
$$

Lemma 1 The values $x_{j}^{i}$ satisfy (4) and (5) if and only if they are a Nash equilibrium.

Proof. The sets $\left\{x_{j}^{i}\right\}_{j}$, for $i \in\{1,2, \ldots, d\}$, are constrained by (4) to be probability distributions.

We claim that expressions (5) are equivalent to the definition of Nash equilibrium constraints (1). If $a_{j}$ give player $i$ a higher payoff that all previous (weaker) strategies then none of those may be in player $i$ 's support. Similarly, when $a_{j}$ gives a lower payoff than a weaker strategy, $a_{j}$ will not be in the support. Note that if $a_{j}$ gives a higher payoff than the weaker actions but a lower payoff than a stronger strategy $a_{j^{\prime}}$ then the probability of playing $a_{j}$ will be set to 0 when $\pi_{j^{\prime}}^{i}$ is compared with $\max _{k=1, \ldots, j, \ldots, j^{\prime}-1}\left\{\pi_{k}^{i}\right\}$.

Consequently we have reduced the problem to satisfying the constraints (44) and (5). We now define variables in addition to $x$ 's and $\pi$ 's with the aim of expressing (44) and (5) in terms of a constant number of "local" variables. This is needed to define our FPTAS. Let $\sigma_{j}^{i}$ be the partial $\operatorname{sum} \sum_{\ell=1}^{j} x_{\ell}^{i}$. We can now express (44) as follows:

$$
\begin{gathered}
\sigma_{1}^{i}=x_{1}^{i} \quad \begin{array}{l}
0 \leq \sigma_{j}^{i} \leq 1 \\
\sigma_{n}^{i}=1
\end{array} \quad \sigma_{j-1}^{i}+x_{j}^{i}=\sigma_{j}^{i} \\
\leq 1
\end{gathered}
$$

and observing $p_{j}^{i}(-1)=\sigma_{j-1}^{i}, p_{j}^{i}(0)=x_{j}^{i}$ and $p_{j}^{i}(1)=1-\sigma_{j}^{i}$, we can now express $\pi_{j}^{i_{1}}$ in (3) only in terms of variables $\sigma_{j-1}^{i_{2}}, \ldots, \sigma_{j-1}^{i_{d}}, x_{j}^{i_{2}}, \ldots, x_{j}^{i_{d}}, \sigma_{j}^{i_{2}}, \ldots, \sigma_{j}^{i_{d}}$ as

$$
\pi_{j}^{i_{1}}=-c_{j}^{i_{1}}+\sum_{v \in\{0\} \times B^{d-1}} \frac{u_{v(1)+1}+\cdots+u_{q}}{v(0)} \cdot \Psi_{j}^{i_{1}}\left(v, \sigma_{j-1}^{i_{2}}, \ldots, \sigma_{j-1}^{i_{d}}, x_{j}^{i_{2}}, \ldots, x_{j}^{i_{d}}, \sigma_{j}^{i_{2}}, \ldots, \sigma_{j}^{i_{d}}\right),
$$

where function $\Psi_{j}^{i_{1}}(\cdot)$ is the product $\prod_{k=2}^{d} p_{j}^{i_{k}}\left(v_{k}\right)$ written in terms of these variables. Observe that if $j=1$, then $\sigma_{j-1}^{i_{2}}=\cdots=\sigma_{j-1}^{i_{d}}=0$, and if $j=n$, then $\sigma_{j}^{i_{2}}=\cdots=\sigma_{j}^{i_{d}}=1$; thus, in these cases function $\Psi_{j}^{i_{1}}(\cdot)$ does not depend on these respective variables.

Additionally, let $\alpha_{j}^{i}$ be the maximum expected payoff player $i$ can get by playing one of $a_{1}, \ldots, a_{j}$, i.e., $\alpha_{j}^{i}=\max _{k=1, \ldots, j}\left\{\pi_{k}^{i}\right\}$. We can now define

$$
\alpha_{1}^{i}=\pi_{1}^{i} \quad \alpha_{j}^{i}=\max \left\{\alpha_{j-1}^{i}, \pi_{j}^{i}\right\}
$$

and express (5) as follows:

$$
\begin{aligned}
& \pi_{j}^{i}>\alpha_{j-1}^{i} \Longrightarrow \sigma_{j-1}^{i}=0 \\
& \pi_{j}^{i}<\alpha_{j-1}^{i} \Longrightarrow x_{j}^{i}=0 .
\end{aligned}
$$

Observation 4 The values $x_{j}^{i}, \sigma_{j}^{i}, \alpha_{j}^{i}$ and $\pi_{j}^{i}$ satisfy (6, 7], 8 ) if and only if the values $x_{j}^{i}$ are $a$ Nash equilibrium.

Now consider the sequence

$$
\mathcal{S}=\left(\pi_{j}^{1}, \ldots, \pi_{j}^{d}, x_{j}^{1}, \ldots, x_{j}^{d}, \alpha_{j}^{1}, \ldots, \alpha_{j}^{d}, \sigma_{j}^{1}, \ldots, \sigma_{j}^{d}\right)_{j=1, \ldots, n} .
$$


Constraints in (6) involve 3 variables that are at distance at most $4 d+1$ in $\mathcal{S}$ (namely, for $j>1$, $\sigma_{j-1}^{i}$ is followed by $4 d$ elements of $\mathcal{S}$-including $x_{j}^{i}$ - and then by $\sigma_{j}^{i}$ ). Constraints (77) on the other hand involve variables that are at distance at most $5 d$ in $\mathcal{S}$. It is easy to check that the same happens also for the other constraints and conclude then that the following holds.

Observation 5 For any $j=1, \ldots, n$, for each constraint in (6), (7), (8) and (9) there are $5 d$ consecutive elements of $\mathcal{S}$ that contains the quantities involved in the constraint.

The algorithm. For $\epsilon>0$ according to (2) we relax the constraints of (9) as follows:

$$
\begin{aligned}
& \pi_{j}^{i}>\alpha_{j-1}^{i}+\epsilon \quad \Longrightarrow \quad \sigma_{j-1}^{i}=0, \\
& \pi_{j}^{i}<\alpha_{j-1}^{i}-\epsilon \quad x_{j}^{i}=0 .
\end{aligned}
$$

Let $\mathcal{S}_{i}$ be the sequence of $5 d$ consecutive elements of $\mathcal{S}$ that begins at the $i$-th element of $\mathcal{S}$. Let $\mathcal{E}_{i}$ be the set of expressions in (66), (7), (8) and (10) that relate elements of $\mathcal{S}_{i}$ with each other; by Observation 5 the union of the sets $\mathcal{E}_{i}$ is all constraints (66), (7), (8) and (10). The algorithm (Algorithm 2) works its way through the sequence $\mathcal{S}$ left-to-right, and for each $\mathcal{S}_{i}$ identifies a subset of $([0,1])^{5 d}$ representing possible values of those quantities that form part of an approximate Nash equilibrium. We call this subset $D_{i}$. Then it sweeps through the sequence right-to-left identifying allowable values for previous elements. The parameter $\epsilon$ controls quality of approximation.

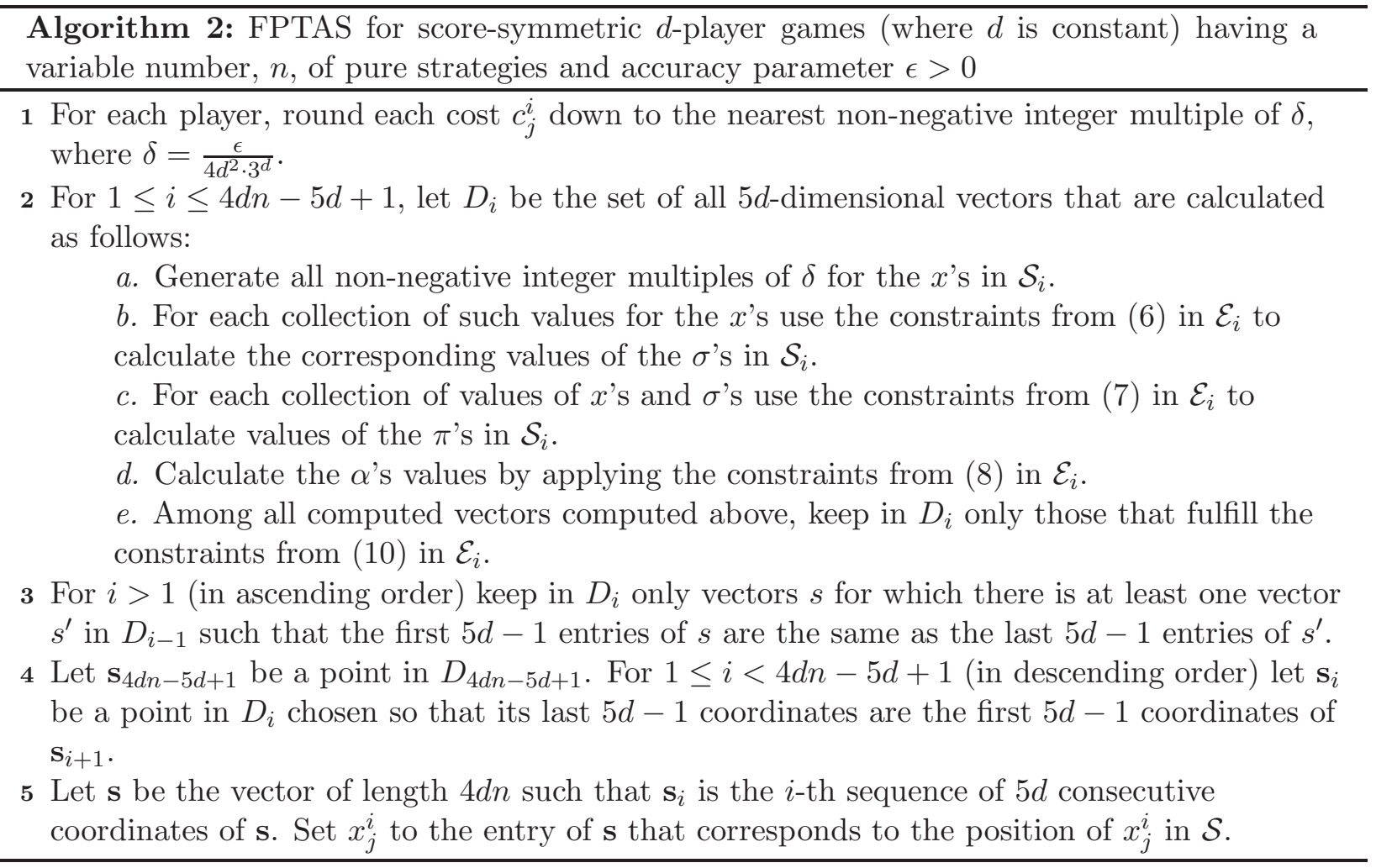

Theorem 7 There is a FPTAS for computing $\epsilon$-Nash equilibria of competitiveness-based ranking games with a constant number of players.

When the number of players is a constant, we may assume that the games is score-symmetric by applying the reduction of Section 3.4.1, which is then achieved at the price of a constant-factor 
increase in the number $n$ of strategies per player. We show that Algorithm 2 is indeed a FPTAS for this class of games. The proofs assume that $\epsilon$ is the inverse of an integer. (Similarly to above, if this is not the case we simply run the algorithm with an $\epsilon^{\prime}<\epsilon$ which is inverse of an integer.) Theorem 7 will follow from the following two propositions and the subsequent observation about the runtime.

Proposition 1 (Approximation guarantee) If Algorithm 2 finds a vector $\mathbf{s}$ in Step 5 , then the values $x_{j}^{i}$ correspond to a $(n+2) \epsilon$-Nash equilibrium.

Proof. The entries of $\mathbf{s}$ (including the subset corresponding to $x_{j}^{i}$ ) satisfy (66), (77), (8) and (10), where (10) simply rewrites the definition of $\epsilon$-Nash equilibrium (2) thus implying that we are losing an additive $\epsilon$. Another additive loss smaller than $\epsilon$ is due to the cost rounding. Furthermore, we are restricting to probability distributions whose values are non-negative integer multiples of $\delta$. Thus, a player may be forced to play a strategy with a probability that differs by at most $\delta$ from the probability of his best response. This may impose an additional additive error of $n \delta<n \epsilon$ in the worst case (this is because we have $n$ actions and on each of them the best response is at most $\delta$ different, while the payoffs are upper bounded by 1 ).

\section{Proposition 2 (Correctness) Algorithm Q always finds a vector $\mathbf{s}$ in Step 5 .}

Proof. Consider a Nash equilibrium $\mathcal{N}$ and the associated vector s. Take a $\delta$-rounding $\tilde{x}(\delta=$ $\left.\epsilon /\left(4 d^{2} \cdot 3^{d}\right)\right)$ for each probability vector $x$ in s. Use (6) to define the corresponding rounded vector $\tilde{\sigma}$. Given $\tilde{x}$ and $\tilde{\sigma}$, use (77) and (8) to define values of $\tilde{\pi}$ and $\tilde{\alpha}$, respectively; the newly-obtained vector of rounded values is denoted as $\tilde{\mathbf{s}}$. Observe that $\tilde{\mathbf{s}}$ is considered by the algorithm. It thus suffices to show that such a sequence satisfies all the constraints that the algorithm imposes on the output.

By construction, (6), (77) and (8) are fulfilled. For constraint (10) we show that $\tilde{\pi}_{j}^{i}>\tilde{\alpha}_{j-1}^{i}+\epsilon \Rightarrow$ $\tilde{\sigma}_{j-1}^{i}=0$. (Very similar arguments can be used to show the other condition of (10).)

We will show first that $\left|y_{j}^{i}-\tilde{y}_{j}^{i}\right|<\epsilon / 2$ for $y \in\{\pi, \alpha\}$. We will only give the details for $y=\pi$ as the argument is similar in the other case. Let us first focus on the product $\prod_{k=2}^{d} p_{j}^{i_{k}}\left(v_{k}\right)$ in (3) in which the factors $\sigma_{j-1}^{i_{k}}, 1-\sigma_{j}^{i_{k}}$ and $x_{j}^{i_{k}}$ are involved. Now, the $\tilde{x}^{\prime}$ 's are defined as $\delta$-roundings of the corresponding $x$ 's (according to Observation 11). The quantity $\tilde{\sigma}_{j}^{i}$ is the sum of the first $j$ entries of $\tilde{x}^{i}$. Thus, Observation 1 allows us to deduce that the rounded values $\tilde{\sigma}_{j-1}^{i_{k}}, 1-\tilde{\sigma}_{j}^{i_{k}}$ and $\tilde{x}_{j}^{i_{k}}$ are less than $\delta$ away from $\sigma_{j-1}^{i_{k}}, 1-\sigma_{j}^{i_{k}}$ and $x_{j}^{i_{k}}$ in absolute value, respectively. In other words, $\left|\tilde{p}_{j}^{i_{k}}\left(v_{k}\right)-p_{j}^{i_{k}}\left(v_{k}\right)\right|<\delta$ for any $i_{k}$ and $j$.

Then we have

$$
\prod_{k=2}^{d} \tilde{p}_{j}^{i_{k}}\left(v_{k}\right)-\prod_{k=2}^{d} p_{j}^{i_{k}}\left(v_{k}\right)<\prod_{k=2}^{d}\left(p_{j}^{i_{k}}\left(v_{k}\right)+\delta\right)-\prod_{k=2}^{d} p_{j}^{i_{k}}\left(v_{k}\right) .
$$

An upper bound for the right-hand side is obtained by setting $p_{j}^{i_{k}}\left(v_{k}\right)=1$, resulting in an upper bound of $(1+\delta)^{d-1}-1$. $\delta$ was chosen sufficiently small to ensure that this is at most $\epsilon /\left(4 \cdot 3^{d-1}\right)$. Now observe that the component $\frac{u_{v(1)+1}+u_{v(1)+2}+\cdots+u_{p}}{v(0)}$ in (3) has value at most one and there are at most $3^{d-1}$ terms in the summation of (3), which implies that the difference between the summations for $\pi_{j}^{i_{1}}$ and $\tilde{\pi}_{j}^{i_{1}}$ is strictly smaller than $\epsilon / 4$. We have $\left|\pi_{j}^{i_{1}}-\tilde{\pi}_{j}^{i_{1}}\right|<\epsilon / 2$.

Thus we have shown that $\left|y_{j}^{i}-\tilde{y}_{j}^{i}\right|<\epsilon / 2$ for $y \in\{\pi, \alpha\}$ and so $-\epsilon / 2<y_{j}^{i}-\tilde{y}_{j}^{i}<\epsilon / 2$ for $y \in\{\pi, \alpha\}$. Therefore $\tilde{\pi}_{j}^{i}>\tilde{\alpha}_{j-1}^{i}+\epsilon$ implies that $\pi_{j}^{i}>\alpha_{j-1}^{i}$ and as $\mathbf{s}$ is a Nash equilibrium, 
by Observation 4 and (9), we have $\sigma_{j-1}^{i}=0$. But then by the way we define $\tilde{\sigma}$ 's we have that $\tilde{\sigma}_{j-1}^{i}=0$.

Runtime. Since the values of $\sigma$ 's, $\pi$ 's and $\alpha$ 's are calculated applying (16), (17) and (8) respectively for given $x$ 's, the sets $D_{i}$ are of size $O\left((1 / \delta)^{d}\right)=O\left((1 / \epsilon)^{d}\right)$, so for constant $d$ the runtime of the algorithm is indeed polynomial in $n$ and $1 / \epsilon$, as required for a FPTAS.

Remarks. Algorithm 2 is somewhat similar to the algorithm of [24] for solving tree-structured graphical games. They give a similar forward-and-backward dynamic programming approach to solving these games; their algorithm takes exponential time for exact equilibria [17] but a similar quantisation of real-valued payoffs leads to a FPTAS.

\section{Conclusions and further work}

Our FPTAS can be used to compute exact equilibria in certain cases. When a game with constantlymany players has payoffs that are multiples of some $\epsilon>0$ then we can compute exact Nash equilibria in time polynomial in the size of the input and $1 / \epsilon$ by simply using the FPTAS. This observation raises the open problem of determining whether there is a polynomial-time algorithm for solving 2-player (competitiveness-based ranking) games in general when ties are possible and the prize is shared in the event of a tie.

Several other concrete open problems have been raised by the current results, for example, fully quantifying the complexity of computing Nash equilibria for competitiveness-based ranking games. Also, in situations where multiple equilibria may exist, we would like to know whether a specific equilibrium is selected by some natural decentralized dynamic process.

Acknowledgements. We thank David Gill, Milan Vojnovik and Yoram Bachrach for pointers to related work.

\section{References}

[1] M. R. Baye, D. Kovenock and C. G. de Vries. The all-pay auction with complete information. Economic Theory, 8, pp. 362-380, 1996.

[2] M. Blonski. Anonymous Games with Binary Actions. Games and Economic Behavior 28(2), pp. 171-80, 1999.

[3] F. Brandt, F. Fischer, P. Harrenstein and Y. Shoham. Ranking Games. Artificial Intelligence 173(3), pp. 221-239, 2009.

[4] F. Brandt, F. Fischer and M. Holzer. Symmetries and the complexity of pure Nash equilibrium. Journal of Computer and System Sciences 75(3), pp. 163-177, 2009.

[5] P. Briest, P.W. Goldberg and H. Röglin. Approximate Equilibria in Games with Few Players. ArXiv report, CoRR abs/0804.4524, 2008.

[6] I. Brochas and J.D. Carrillo. Do the "three-point victory" and "golden goal" rules make soccer more exciting? Journal of Sports Economics, 5, pp. 169-185, 2004. 
[7] W. Chan, P. Courty and L. Hao. Suspense: Dynamic Incentives in Sports Contests. The Economic Journal, 119, pp. 24-46, 2009.

[8] Y.-K. Che and I. Gale. Optimal Design of Research Contests. American Economic Review 93(3) pp. 646-671, 2003.

[9] X. Chen, X. Deng and S.-H. Teng, Settling the Complexity of Computing Two-Player Nash Equilibria. Journal of the ACM 56(3), 2009.

[10] S.-F. Cheng, D.M. Reeves, Y. Vorobeychik and M.P. Wellman. Notes on equilibria in symmetric games. Proc. 6th International Workshop on Game Theoretic and Decision Theoretic Agents (GTDT), pp. 23-29, 2004.

[11] C. Cohen, T. R. Kaplan, A. Sela. Optimal rewards in contests. RAND Journal of Economics, 39(2), 434-451, 2008.

[12] C. Daskalakis. An Efficient PTAS for Two-Strategy Anonymous Games. Proc. 4th Workshop on Internet $\& 5$ Network Economics (WINE), pp. 186-197, 2008.

[13] C. Daskalakis, P.W. Goldberg and C.H. Papadimitriou. The Complexity of Computing a Nash Equilibrium. SIAM Journal on Computing 39(1), pp. 195-259, 2009.

[14] C. Daskalakis and C.H. Papadimitriou. Discretized Multinomial Distributions and Nash Equilibria in Anonymous Games. Proc. of 49th Annual IEEE Symposium on Foundations of Computer Science (FOCS), pp. 25-34, 2008.

[15] C. Daskalakis and C.H. Papadimitriou. On a Network Generalization of the Minmax Theorem. Proc. of 36th International Colloquium on Automata, Languages and Programming (ICALP), pp. 423-434, 2009.

[16] C. Daskalakis and C.H. Papadimitriou. On Oblivious PTAS's for Nash Equilibrium. Proc. of 41st ACM Symposium On Theory of Computing (STOC), pp. 75-84, 2009.

[17] E. Elkind, L.A. Goldberg and P.W. Goldberg. Nash Equilibria in Graphical Games on Trees Revisited. Proc. 7th ACM Conference on Electronic Commerce (ACM EC), pp. 100-109, 2006.

[18] J. Fearnley, P.W. Goldberg, R. Savani and T.B. Sørensen. Approximate Well-Supported Nash Equilibrium Below Two-Thirds. Proc. of the 5th Symp. on Algorithmic Game Theory, LNCS 7615, pp. 108-119, 2012.

[19] L.A. Goldberg, P.W. Goldberg, P. Krysta and C. Ventre. Ranking Games that have Competitiveness-based Strategies. Proc. 11th ACM Conference on Electronic Commerce (ACM EC), pp. 335-344, 2010.

[20] K.K. Haugen. Point Score Systems and Competitive Imbalance in Professional Soccer. Journal of Sports Economics 9(2), pp. 191-210, 2008.

[21] S. Hémon, M. de Rougemont and M. Santha. Approximate Nash Equilibria for Multi-player Games. 1st Symposium on Algorithmic Game Theory, pp. 267-278, 2008.

[22] A. Hillman and J. G. Riley. Politically contestable rents and transfers. Economics and Politics. 1, pp. 17-40, 1989. 
[23] E. Hopkins and T. Kornienko. Running to Keep in the Same Place: Consumer Choices as a Game of Status. American Economic Review 94(4), pp. 1085-1107, 2004.

[24] M. Kearns, M. Littman and S. Singh. Graphical Models for Game Theory. Proc. of the 17th Conference in Uncertainty in Artificial Intelligence (UAI), pp. 253-260, 2001.

[25] K.A. Konrad. Strategy and Dynamics in Contests. Oxford University Press, 2009. Early draft available as WZB-Markets and Politics Working Paper No. SP II 2007-01.

[26] E.P. Lazear and S. Rosen. Rank-Order Tournaments and Optimal Labor Contracts. The Journal of Political Economy, 89(5) pp. 841-864, 1981.

[27] B. Moldovanu and A. Sela. The Optimal Allocation of Prizes in Contests. The American Economic Review, 91 (3), pp. 542-558, 2001.

[28] B. Moldovanu and A. Sela. Contest Architecture. Journal of Economic Theory, 126, pp. 70-96, 2006.

[29] B. Moldovanu, A. Sela and X. Shi. Contests for Status. Journal of Political Economy, 115(2), pp. 338-363, 2007.

[30] J. Nash. Noncooperative Games. Annals of Mathematics 54, pp. 289-295, 1951.

[31] N. Nisan, T. Roughgarden, É. Tardos, and V. Vazirani (eds.). Algorithmic Game Theory. Cambridge university press, 2007.

[32] M.J. Osborne and A. Rubinstein. A Course in Game Theory, MIT Press, 1994.

[33] R. Siegel. All-pay contests. Econometrica 77(1), pp. 71-92, 2009.

[34] S. Szymanski. The Economic Design of Sporting Contests. Journal of Economic Literature 41 pp. 1137-87, 2003.

[35] H. Tsaknakis and P.G. Spirakis. An Optimization Approach for Approximate Nash Equilibria. Internet Mathematics 5(4), pp. 365-382, 2008.

[36] G. Tullock. Efficient rent-seeking. In: Buchanan, J.M., Tollison, R.D., Tullock, G. (Eds.), Toward a Theory of Rent-Seeking Society. Texas A\&M University Press, College Station. pp. 97112, 1980. 